\title{
The Effect of Paramagnetic Contrast in Choline Peak in Patients with Glioblastoma Multiforme Might Not Be Significant
}

\author{
E.C. Lima, M.C.G. Otaduy, M. Tsunemi, R. Pincerato, E.F. Cardoso, S. Rosemberg, P.H. Aguiar, G.G. Cerri, and C.C. Leite
}

\begin{abstract}
BACKGROUND AND PURPOSE: ' $\mathrm{H}-\mathrm{MR}$ spectroscopy is a useful tool in brain tumor evaluation. A critical point in obtaining representative spectra is the correct voxel positioning, which can be more accurate after Gd administration. Some experimental data suggested that Gd could cause Cho signal loss. Our aim was to evaluate the effect of Gd in the Cho peak area and width in patients with GBM.
\end{abstract}

MATERIALS AND METHODS: We performed multivoxel ' $\mathrm{H}-\mathrm{MR}$ spectroscopy before and after Gd administration in 18 patients with GBM. Quantification of Cho peak area and width in each voxel was completed, and the Cho mean and maximum values before and after Gd injection were calculated in the tumor and contralateral hemisphere. Choline peak area and width values obtained before and after contrast were compared, considering as separate entities enhancing and nonenhancing tumoral voxels and the contralateral hemisphere.

RESULTS: No statistically significant differences were found for the Cho peak area mean values in the tumoral voxels or contralaterally $(P>$.05). A tendency for an increase in the Cho peak width mean value was found in the tumoral enhancing voxels $(P=.055)$. A statistically significant decrease was found for the mean value of the maximum Cho peak area in enhancing tumoral voxels $(P=.020)$. No significant differences were found in the nonenhancing tumoral voxels or contralaterally $(P>.05)$.

CONCLUSIONS: The injection of Gd before performing ' $\mathrm{H}-\mathrm{MR}$ spectroscopy might not significantly affect the Cho peak area in patients with GBM. The paramagnetic contrast seems to cause a different effect, depending on Gd enhancement.

ABBREVIATIONS: Gd = gadolinium; GD-DTPA = gadopentetate dimeglumine; GD-DTPA-BMA = gadodiamide; GBM = glioblastoma multiforme; GD-DOTA = gadoterate meglumine

C onventional MR imaging is the most useful radiologic technique in the detection of intracranial tumors. However, depending on the histologic type of the tumor, the diagnosis is correctly made in only $30-60 \%$ of cases. ${ }^{1}$

In vivo ${ }^{1} \mathrm{H}-\mathrm{MR}$ spectroscopy allows noninvasive evaluation of tumor metabolic profile, and it is useful in the diagnosis of brain tumors as well as in the characterization of metabolic changes associated with tumor progression, degree of malignancy, and treatment response. ${ }^{2-8}$ More recently, it has been shown that ${ }^{1} \mathrm{H}$-MR spectroscopy may also be useful in targeting stereotactic biopsies and planning radiation therapy. ${ }^{3,8}$

A critical point in obtaining representative spectra is the cor-

Received February 2, 2012; accepted after revision March 21. From the Departments of Radiology (E.C.L., M.C.G.O., M.T., R.P., E.F.C., G.G.C., C.C.L.), Pathology (S.R.), and Neurosurgery (P.H.A.), University of Sao Paulo, Sao Paulo, Brazil.

Please address correspondence to Eduardo Carneiro Lima, MD, Hospital Israelita Albert Einstein, Radiologia, Avenida Albert Einstein, 627-Morumbi Departamento de Radiologia, Bloco D $4^{\circ}$ Andar, Sao Paulo 05652900, Brazil; e-mail: eduardocl@einstein.br

http://dx.doi.org/10.3174/ajnr.A3181 rect voxel positioning in the most representative and solid portion of the tumor. Paramagnetic contrast administration may be useful in order to better select the most solid and representative area of the tumor, particularly in heterogeneous lesions with necrotic components. There are, however, some experimental data suggesting that ${ }^{1} \mathrm{H}-\mathrm{MR}$ spectroscopy should be performed before contrast administration, as the paramagnetic contrast could cause loss of the Cho signal, the major metabolic tumor marker. ${ }^{9-11}$ Therefore, this concept is still controversial in the literature. Some authors did not find any significant effect of the paramagnetic contrast in the tumor spectra, while others did find a significant effect, but the techniques used varied substantially. ${ }^{12}$

The aim of this study was to determine whether the administration of the paramagnetic contrast affects the Cho peak area and width obtained by using multivoxel spectroscopy with a long echo time in patients with glioblastoma multiforme.

\section{MATERIALS AND METHODS Subjects}

We evaluated 18 patients with nontreated glioblastoma multiforme, 10 men and 8 women, ranging in age from 43-83 years (mean age, 


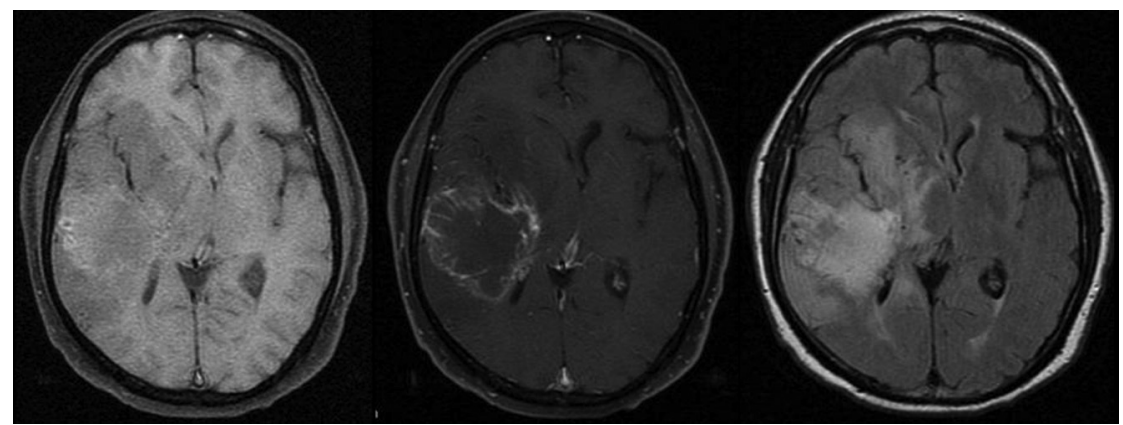

FIG 1. Patient 1. Glioblastoma multiforme: $\mathrm{Tl}$ before and after IV contrast injection and FLAIR before contrast.

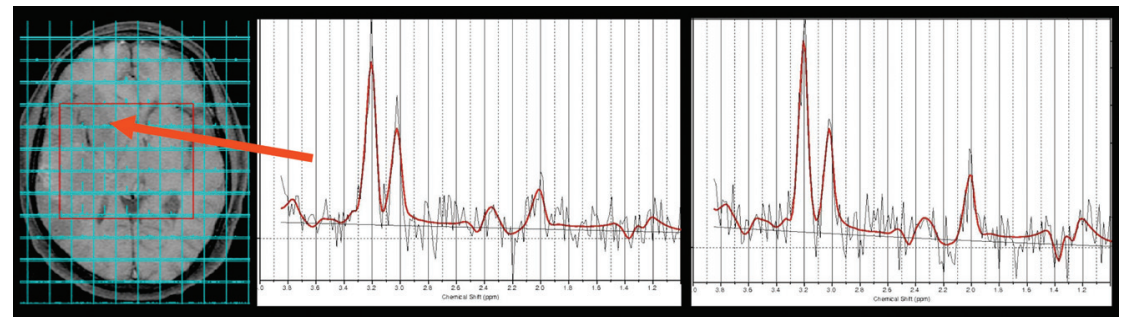

FIG 2. Patient 1. Multivoxel ${ }^{1} \mathrm{H}-\mathrm{MR}$ spectroscopy covering the largest area of the tumor and the spectra obtained before and after IV contrast injection in the medial margin of the tumor.

of interest selected were located on different tumor solid locations, avoiding necrotic areas, and in normal-appearing brain in the contralateral hemisphere. Voxels with negligible signal were not included in the study. Quantification of the individual spectra was performed with LCModel software (Stephen Provencher, Oakville, Ontario, Canada). For each patient, mean Cho peak area and mean Cho peak width values before and after Gd injection were calculated, considering enhancing and nonenhancing tumoral voxels and the contralateral hemisphere separately. We also calculated mean Cho peak area and mean Cho peak width values for the voxels of maximum Cho peak area in the enhancing and nonenhancing tumoral areas and in the contralateral hemisphere of each patient. For a given patient, the number of tumoral voxels analyzed could vary from 1-10 depending on the tumor size and MR spectroscopy quantification viability.

Results of Cho peak area and width values considering the mean and maxi-

63.2 years). This protocol was approved by our institutional review board. All the patients were enrolled in this study after giving informed consent.

\section{MR Imaging/MR Spectroscopy}

All patients underwent MR imaging and multivoxel ${ }^{1} \mathrm{H}-\mathrm{MR}$ spectroscopy on 1.5T equipment (Horizon LX 8.3 scanner; GE Healthcare, Milwaukee, Wisconsin) using the standard quadrature head coil. MR imaging included an axial T1-weighted spin-echo sequence (TR/TE: 466/14 ms), an axial T2-weighted fast spin-echo sequence (TR/TE: 5100/102 ms), an axial FLAIR sequence (TR/TE/TI: 10,002/ 110/2200 ms), and multiplanar T1-weighted spin-echo sequences after the paramagnetic contrast injection (Fig 1). Multivoxel ${ }^{1} \mathrm{H}-\mathrm{MR}$ spectroscopy was performed covering the largest area of T1/T2 abnormality at the site of the tumor using point-resolved spectroscopy (TR/TE: 1500/135 ms) before and 6-7 minutes after Gd injection $(0.1 \mathrm{mmol} / \mathrm{Kg})$ (Fig 2). The contrast agent used varied randomly between GD-DTPA, GD-DOTA, and GD-DTPA-BMA during the study. The chemical shift imaging sequence produced a $16 \times 16$ transversely oriented matrix that was defined by phase encoding with a field of view of $24 \times 24 \mathrm{~cm}$, resulting in an individual voxel size of $15 \times 15 \times 10 \mathrm{~mm}$. After automated transmitter and receiver adjustment, the signal intensity over the volume of interest was shimmed within a full width at half maximum of 0.07 to $0.17 \mathrm{ppm}$. Water resonance suppression was achieved applying chemical shift selective suppression pulses. Contralateral normal tissue was also included as a reference for postprocessing of metabolites and region definition.

\section{Quantification of Metabolite Ratios and Statistical Methods}

${ }^{1} \mathrm{H}$-MRS data were processed off-line with the software SAGE (GE Healthcare) to select the individual spectra of interest. The voxels mum Cho values for each patient before and after contrast injection were compared using the paired $t$ test for the Cho peak area and the Wilcoxon test for the Cho peak width to establish if there were statistically significant differences between the results, considering $P<.05$ to be significant.

\section{RESULTS}

For the same patient, the number of tumoral voxels analyzed varied from $1-10$, resulting in a total of 64 voxels for the 18 patients, distributed as follows: 1 voxel in 4 patients, 2 voxels in 4 patients, 3 voxels in 3 patients, 4 voxels in 3 patients, 5 voxels in 1 patient, 7 voxels in 1 patient, 9 voxels in 1 patient, and 10 voxels in 1 patient. All patients $(n=18)$ presented voxels with some degree of enhancement. A total of 49 voxels with Gd enhancing tumors were included in the analysis. Voxels with nonenhancing tumors were present only in 10 of the 18 patients, resulting in a total of 15 analyzed tumor voxels with no detectable enhancement. In the contralateral normal hemisphere, 32 voxels were analyzed as control voxels. Results of the Cho mean and maximum values are presented in Tables 1 and 2. Other metabolites, such as $\mathrm{N}$-acetylaspartate or creatine, were not observed, as the concentration was too small (under detection limits) for most of the tumor voxels.

No statistically significant differences were found considering the mean value for the Cho peak area per patient before and after venous contrast injection in the enhancing and nonenhancing tumoral voxels, and in the contralateral hemisphere (control), as shown in Table 1 . There was only a tendency $(P=.054)$ for an increase of Cho peak mean value after Gd injection in the nonenhancing tumoral voxels, with mean values of $472 \pm 139$ and $515 \pm$ 160 before and after contrast, respectively. 
Table 1: Mean values for the Cho peak distributed in the tumoral and contralateral normal areas and in the enhancing and nonenhancing tumoral voxels

\begin{tabular}{lcccccccc}
\hline & S & N & Cho Pre & Cho Post & $\boldsymbol{P}$ & LW Pre & LW Post & $\boldsymbol{P}$ \\
\hline Normal contralateral & 18 & 32 & $408 \pm 108$ & $384 \pm 104$ & 0.154 & $0.079 \pm 0.021$ & $0.084 \pm 0.026$ & 0.106 \\
Nonenhanced voxels & 10 & 15 & $472 \pm 139$ & $515 \pm 160$ & 0.054 & $0.081 \pm 0.017$ & $0.095 \pm 0.024$ & 0.119 \\
Enhanced voxels & 18 & 49 & $500 \pm 106$ & $487 \pm 108$ & 0.510 & $0.090 \pm 0.019$ & $0.099 \pm 0.028$ & 0.055 \\
\hline
\end{tabular}

Note:- LW indicates line width; N, total number of voxels analyzed; S, number of subjects analyzed; Pre, precontrast; Post, postcontrast.

Table 2: Mean values for the Cho peak maximum values distributed in the tumoral and contralateral normal areas and in the enhancing and nonenhancing tumoral voxels

\begin{tabular}{lcccccccc}
\hline & S & N & Cho Pre & Cho Post & $\boldsymbol{P}$ & LW Pre & LW Post & $\boldsymbol{P}$ \\
\hline Normal contralateral & 18 & 32 & $430 \pm 112$ & $402 \pm 100$ & 0.148 & $0.081 \pm 0.026$ & $0.085 \pm 0.034$ & 0.154 \\
Non enhanced voxels & 10 & 15 & $489 \pm 159$ & $533 \pm 175$ & 0.077 & $0.084 \pm 0.018$ & $0.095 \pm 0.028$ & 0.172 \\
Enhanced voxels & 18 & 49 & $599 \pm 170$ & $527 \pm 201$ & $0.020^{*}$ & $0.092 \pm 0.025$ & $0.093 \pm 0.027$ & 0.688 \\
\hline
\end{tabular}

Note:- LW indicates line width; N, total number of voxels analyzed; S, number of subjects analyzed; Pre, precontrast; Post, postcontrast; *, statistically significant.

No statistically significant differences were found considering the mean value for the Cho peak width per patient before and after venous contrast injection in the enhancing and nonenhancing tumoral voxels, nor in the contralateral hemisphere, as shown in Table 1. Only in the enhancing tumoral voxels could we observe a tendency $(P=.055)$ toward line broadening after Gd injection, with mean values of $0.090 \pm 0.019$ and $0.099 \pm 0.028$ before and after contrast, respectively.

Considering the maximum Cho peak value for each patient (Table 2), a statistically significant decrease was noted for the enhancing tumoral voxels: $599 \pm 170$ before contrast and $527 \pm$ 201 after contrast, $P=.020$. On the contrary, the Cho maximum value of nonenhancing tumoral voxels presented an increase in Cho, with $489 \pm 159$ and $533 \pm 175$ before and after contrast, respectively, though this increase did not reach statistical significance $(P=.077)$. No significant differences were found in the contralateral hemisphere, with $430 \pm 112$ and $402 \pm 100$ before and after contrast, respectively $(P=0.148)$.

The Cho peak width, when considering only those voxels of maximum Cho peak area, did not change significantly with Gd injection in any of the analyzed regions (Table 2).

\section{DISCUSSION}

MR spectroscopy has become a useful tool in the characterization and evaluation of brain tumors. MR spectra of brain tumors usually show elevated Cho levels and decreased levels of $\mathrm{N}$-acetylaspartate, reflecting increased cellular membrane turnover and neuronal cell dysfunction/death, respectively. ${ }^{1-6,8,13-15}$ Nelson ${ }^{8}$ showed that the relative increase in Cho and decrease in NAA is critical for defining the spatial extent of the metabolic abnormality corresponding to an active tumor. Nelson developed an index for clinical applications, termed Cho-to-NAA index that, based on its value, assigns a probability value of tumor presence. Fountas et $\mathrm{al}^{16}$ showed that the higher the grade of the astrocytomas, the higher the Cho peak and the lower the Cr peak. They were able to differentiate between astrocytoma grades II, III and IV by using the $\mathrm{Cho} / \mathrm{Cr}$ ratio, and proposed considering this ratio as a malignant index for the histologic grading of these tumors.

The use of the paramagnetic contrast before the acquisition of the spectroscopy may aid voxel positioning in the most representative and solid portion of the tumor, but some experimental studies have suggested that Gd-contrast could lead to loss of the Cho signal, leading to errors in tumor evaluation. ${ }^{10,11}$ Therefore, it is important to characterize the effect of the paramagnetic contrast on the metabolite values, particularly Cho.

Previous studies have shown that when using T2-weighted spectroscopy (ie, using a long TE), the Cho peak area and line shape are influenced by paramagnetic contrast agents. ${ }^{10,11}$ These 2 studies reported a mean decrease of Cho peak area of $12 \%$ and $15 \%$ when using chemical shift imaging point-resolved spectroscopy (TR/TE: 1500/135 ms).

Although we used the same acquisition technique in our study, we did not find statistically significant differences before and after contrast in the Cho peak area in the tumor, nor in the contralateral hemisphere, calculating mean values for the enhancing and nonenhancing tumoral voxels and also in the contralateral hemisphere.

The paramagnetic contrast can enhance both longitudinal and transversal metabolite relaxation, leading to effective shorter T1 and T2 relaxation times. A T1 shortening would result in a metabolite intensity gain, especially if the spectrum is acquired with a short TR. On the other hand, a T2 shortening will result in a loss of metabolite intensity, becoming more pronounced the longer the $\mathrm{TE}$ of the acquisition. Based on an in vitro study, Murphy et $\mathrm{a}^{17}$ showed that, for the acquisition parameters TE/TR $=135 / 1500$ $\mathrm{ms}$, the actual contrast concentration in the voxel selected for the ${ }^{1} \mathrm{H}-\mathrm{MR}$ spectroscopy study will determine which of both effects will dominate, that is, whether an increase or decrease of metabolite intensity will be observed. A smaller Gd concentration would predominantly cause an increase in the $\mathrm{T} 1$ relaxation of the Cho, leading to an increase in its signal. At higher concentrations of contrast, transverse relaxation enhancement becomes more significant and metabolite intensity begins to drop. ${ }^{17}$ Although the changes in the mean Cho peak area found in our study were not statistically significant, it is interesting to note that, individually, we found a large variation before and after contrast administration, ranging from negative to positive values, probably depending on the contrast concentration in each voxel as described by Murphy et al. ${ }^{17}$ After contrast administration we observed a mean increase in the Cho peak area in the nonenhancing tumoral voxels, where an increase in the T1 relaxation predominates leading to an increase in the Cho signal and a mean decrease in the Cho peak area in the enhancing tumoral voxels, where T2 relaxation becomes more pronounced leading to a decrease in the Cho signal. 
We found it interesting to pick the voxel of the highest Cho concentration in each patient and to study the effect of the Gd in this particular voxel, as some authors suggested that this voxel could represent the highest metabolic activity area of the tumor and could be used to classify the degree of the tumoral malignance, and to target stereotatic biopsy and radiation therapy. ${ }^{8}$ When considering the mean values for the maximum Cho peak area value in each patient, a statistically significant decrease was noted in the Cho peak area mean value after contrast injection in the enhancing tumoral voxels where the Gd concentration is higher, and a tendency of increase in the nonenhancing tumoral voxels Cho peak area mean value, though not statistically significant $(P=.077)$, where the Gd concentration is lower.

Other factors that could have contributed to this large variation include the heterogeneity of the GBM itself, the variability inherent in the method, and the type of Gd-contrast agent used. The large variation in voxel values involving the Cho peak area before and after contrast injection observed in our study might indicate that is advisable to consider several voxels within the tumor, instead of choosing just one to calculate the values for Cho when IV contrast injection is performed before data acquisition, particularly when analyzing heterogeneous contrast-enhancing tumors such as glioblastoma multiforme.

One factor that is important to consider when studying paramagnetic contrast interaction with brain metabolites is cellular compartmentalization. The cell membrane inhibits intracellular metabolites from coming into contact with extracellular contrast agents. Because of their charge and structure, they do not freely pass through the intact cell membrane. 9,18,19 This means that for the contrast agents to interact directly with the metabolites, the metabolites should be in the extracellular compartment. Sijens et $\mathrm{al}^{10}$ consider that there is a pool of extracellular Cho that is increased in brain tumors and interacts with the paramagnetic contrast, leading to a dipolar relaxation with loss of signal of this metabolite. In the dipolar relaxation there is a dipole-dipole interaction between the protons of the metabolite and the unpaired electrons of the Gd, which is responsible for the T1 and T2 shortening of the metabolites. This would explain why the effect of Gd is more appreciable on the Cho signal, and also present in the extracellular space, than on other metabolites.

Theoretically, contrast agents could also affect the measured MR signal indirectly by inducing $\mathrm{T} 2{ }^{\star}$ susceptibility derived relaxation of intracellular metabolite from the extracellular space. ${ }^{9} \mathrm{~A}$ nonhomogeneous magnetic field within the volume of interest leads to slight differences in the precession frequency of the hydrogen protons within the same molecule, increasing the metabolite line width, which could cause loss of the signal-to-noise ratio and overlap of the metabolite peaks. ${ }^{18}$ Hence, paramagnetic contrast distribution could create magnetic field perturbation, leading to metabolite peak widening. We calculated the width of the line fitted to the Cho peak before and after contrast administration across the tumoral area and the contralateral hemisphere, considering the mean value per patient and also the width of the Cho peak maximum value voxel in the enhancing and nonenhancing tumoral voxels, and in the contralateral hemisphere in each patient. Although no statistically significant differences were found, a tendency of increase in the Cho peak width mean value was observed when considering the mean values for the Cho peak width in the enhancing tumoral voxels. This suggests that Gd injection really could cause a $\mathrm{T} 2{ }^{*}$ susceptibility effect on the spectrum. This can be important if the Cho signal is estimated only by the measurement of peak amplitudes, because $\mathrm{T} 2{ }^{\star}$ line broadening leads to lower peak heights.

A limitation of our study was that we could not control which Gd complex was to be used. The properties of the contrast agent itself are likely to influence the efficacy of paramagnetic relaxation of metabolite protons. These differences may occur due to different complex charges and/or different chelate structures, which would prevent or facilitate the Gd coming closer to the metabolite protons. The 3 more commonly used contrast agents, which were also used in our study-GD-DTPA, GD-DOTA, and GD-DTPABMA- have different charges and molecular structure. GDDTPA and GD-DTPA-BMA are structurally analogous, though they possess different overall charges, -2 in GD-DTPA and 0 in GD-DTPA-BMA. The complex GD-DOTA has an overall charge of -1 but has a macrocyclic structure, which makes a direct interaction much more difficult compared with the open structures of DTPA and DTPA-BMA. ${ }^{17}$ In an in vitro study, Murphy et al ${ }^{17}$ found that these $3 \mathrm{Gd}$ agents are all able to efficiently enhance the relaxation of the Cho methyl protons, which have a positive overall charge, with GD-DTPA being more efficient in enhancing the relaxation of Cho than the other 2 paramagnetic complexes, with a clear relationship between relaxivity and the ionic charge of the metabolite and of the contrast agent.

\section{CONCLUSIONS}

The injection of paramagnetic contrast before performing multivoxel MR spectroscopy might not significantly affect the estimated Cho peak area in the diagnosis and evaluation of glioblastoma multiforme when considering several tumoral voxels together. However, the effect of Gd on different voxels within a tumor might vary a lot, probably depending on the amount of Gd concentration found in each voxel. Gd seems to cause local magnetic field inhomogeneity, indicated by a tendency of widening of the mean Cho peak width in the tumoral enhancing voxels after contrast administration.

Disclosures: Maria CG Otaduy—UNRELATED: Employment: Fundação da Faculdade de Medicina; Grants/Grants Pending: FAPESP-Fundação de Amparo a Pesquisa do Estado de São Paulo;* Travel/Accommodations/Meeting Expenses Unrelated to Activities Listed: FAPESP — Fundação de Amparo a Pesquisa do Estado de São Paulo. Claudia Leite-UNRELATED: Grants/Grants Pending: Geurbet;* Payment for Lectures (including service on speakers bureaus): Guerbet, Comments: I was invited to be the host of a meeting in Rio de Janeiro sponsored by Guerbet. ( ${ }^{\star}$ Money paid to institution)

\section{REFERENCES}

1. Howe FA, Opstad KS. ${ }^{1}$ HMR spectroscopy of brain tumours and masses. NMR Biomed 2003;16:123-31

2. Law M. MR spectroscopy of brain tumors. Top Magn Reson Imaging 2004;15:291-313

3. Leclerc X, Huisman TA, Sorensen AG. The potential of proton magnetic resonance spectroscopy $\left({ }^{1} \mathrm{H}\right.$-MRS) in the diagnosis and management of patients with brain tumors. Curr Opin Oncol 2002; 14:292-98

4. Majós C, Alonso J, Aguilera C, et al. Proton magnetic resonance spectroscopy $\left({ }^{1} \mathrm{H}\right.$ MRS) of human brain tumours: assessment of 
differences between tumour types and its applicability in brain tumour categorization. Eur Radiol 2003;13:582-91

5. McKnight TR. Proton magnetic resonance spectroscopic evaluation of brain tumor metabolism. Semin Oncol 2004;31:605-17

6. Möller-Hartmann W, Herminghaus S, Krings T, et al. Clinical application of proton magnetic resonance spectroscopy in the diagnosis of intracranial mass lesions. Neuroradiology 2002;44:371-81

7. Murphy M, Loosemore A, Clifton AG, et al. The contribution of proton magnetic resonance spectroscopy $\left({ }^{1} \mathrm{HMRS}\right)$ to clinical brain tumour diagnosis. Br J Neurosurg 2002;16:329-34

8. Nelson SJ. Multivoxel magnetic resonance spectroscopy of brain tumors. Mol Cancer Ther 2003;2:497-507

9. Murphy PS, Dzik-Jurasz AS, Leach MO, et al. The effect of Gd-DTPA on T(1)-weighted choline signal in human brain tumours. Magn Reson Imaging 2002;20:127-30

10. Sijens PE, Oudkerk M, van Dijk P, et al. ${ }^{1} \mathrm{HMR}$ spectroscopy monitoring of changes in choline peak area and line shape after Gd-contrast administration. Magn Reson Imaging 1998;16:1273-80

11. Sijens PE, van den Bent MJ, Nowak PJ, et al. ${ }^{1} \mathrm{H}$ chemical shift imaging reveals loss of brain tumor choline signal after administration of Gd-contrast. Magn Reson Med 1997;37:222-25

12. Smith JK, Kwock L, Castillo M. Effects of contrast material on sin- gle-volume proton MR spectroscopy. AJNR Am J Neuroradiol 2000;21:1084-89

13. Lukas L, Devos A, Suykens JA, et al. Brain tumor classification based on long echo proton MRS signals. Artif Intell Med 2004;31:73-89

14. Maheshwari SR, Fatterpekar GM, Castillo M, et al. Proton MR spectroscopy of the brain. Semin Ultrasound CT MR 2000;21:434-51

15. Meyerand ME, Pipas JM, Mamourian A, et al. Classification of biopsy-confirmed brain tumors using single-voxel MR spectroscopy. AJNR Am J Neuroradiol 1999;20:117-23

16. Fountas KN, Kapsalaki EZ, Vogel RL, et al. Noninvasive histologic grading of solid astrocytomas using proton magnetic resonance spectroscopy. Stereotact Funct Neurosurg 2004;82:90-97

17. Murphy PS, Leach MO, Rowland IJ. Signal modulation in (1) H magnetic resonance spectroscopy using contrast agents: proton relaxivities of choline, creatine, and N-acetylaspartate. Magn Reson Med 1999;42:1155-58

18. Lin AP, Ross BD. Short-echo time proton MR spectroscopy in the presence of gadolinium. J Comput Assist Tomogr 2001;25:705-12

19. Murphy PS, Leach MO, Rowland IJ. The effects of paramagnetic contrast agents on metabolite protons in aqueous solution. Phys Med Biol 2002;47:N53-59 